\begin{tabular}{|c|c|}
\hline ב & $\begin{array}{c}\text { International Journal of Current Research } \\
\text { and Academic Review }\end{array}$ \\
\hline $\mathrm{VT}_{\mathrm{TT}}$ & $\begin{array}{r}\text { ISSN: } 2347-3215 \text { (Online) }, ;, ; \text { Volu } \\
\text { Journal hompage: }\end{array}$ \\
\hline
\end{tabular}

doi: http://dx.doi.org/10.20546/ijcrar.2017.502.002

\title{
Isolation, Diagnosis and Cultivation of Trichomonas gallinae from Domestic Pigeon in Kirkuk City, Iraq
}

\author{
Sadia Sh. Hamad* and Hazha H. Hassan \\ Department of Parasitology, College of Science, University of Kirkuk, Iraq \\ *Corresponding author
}

\section{Abstract}

This research was conducted to study the spread of an important unicellular protozoan Trichomonas gallinae isolated from the mouth and esophagus of three groups of domestic pigeon Colomba livia domestica by wet mount preparation. A total of 609 domestic pigeons were tested in Kirkuk city, Iraq from August 2015 to April 2016, the total infectious rate of the prevalence were $49.26 \%$, distributed to $64.92 \%, 50.98 \%$ and $30.41 \%$ in Alzajel, pakistani and zubaeri pigeon respectively. The present study compared three different stains to characterize the shape of $T$. gallinae namely Giemz, Gram and for first time Field's stain, the Giemza stain showed efficient to stain $T$. gallinae more than other two stains, although Field's stain requires less time for staining compared to Giemza and Gram's stain. The parasites are cultivated in three prepared culture media (In-tube culture system), which are modified Diamond media (TYM), Cysteine Peptone Maltose media (CPLM) and Trichomonas CM0161 media (CM 161) with the initial inoculation of $2 X 105 \mathrm{cell} / \mathrm{ml}$, the fourth media was In-pouch culture system that is commercially available with the initial inoculation of $105 \mathrm{cell} / \mathrm{ml}$. In-pouch TV culture media is used for the first time to diagnose and to grow $T$. gallinae which is a special media for diagnosis of the vaginal trichomoniasis caused by Trichomonas vaginalis. The result showed the best growth of $T$. gallinae in In-pouch media compared with In-tube culture system, modified diamond media and CM 161 gave the high growth to 48 hours while CPLM gave the high growth from 24 hours, the CM 161 media showed acute decrease in the number of $T$. gallinae after 48 hour.
\end{abstract}

\section{Article Info}

Accepted:25 January 2017

Available Online:20 February 2017

\section{Keywords}

Trichomonas gallinae,

Domestic pigeon,

Colomba livia domestica.

\section{Introduction}

Trichomonas gallinae, the etiologic agent of avian trichomoniasis which is a mitochondrial anaerobic protozoan and a flagellated parasite in the class Zoomastigophorea and order Trichomonadida. The protozoa are the world-wide parasitic disease, that found in the superior digestive system of clinically and subclinically infected birds. In clinically infected birds causes pathologic changes, such as inflammation of the mucosa and caseous lesions, which can block the esophagus and subsequently causing death of the host through starvation (Narcisi et al., 1991; Grabensteiner et al., 2010).

Trichomonas gallinae divided by binary fission, resulting in the formation of lesions, mostly in the respiratory tract and esophagus. The Trichomonads has no intermediate 
host but can be transmitted both vertically in columbiformes through pigeon crop milk which is fed to young nestlings and horizontally at shared water and food sources (Bunbury et al., 2007). T. gallinae causes the disease in pigeon called as canker mainly affecting the crop, esophagus, liver and lung. Cankers appear to be a host response to the presence of trichomonads, and are composed of blood cells (primarily leucocytes), tissue debris, degenerate parasites and other components.

Signs of avian trichomoniasis other than cankers and intense inflammation include emaciation, hunched posture, ruffled feathers, closed eyes and gasping for air. Birds near death lose perching and/or standing abilities. T. gallinae is the most pathogenic member of the genus Trichomonas, yet literature concerning its relationship with its hosts during canker formation is scarce. The most significant work on the lesions of this disease was performed by Mesa et al., (1961).

Columbidae are known as the T. gallinae main host, especially the domestic pigeon (Columba livia), which has been believed to be responsible for the world-wide prevalence of parasite (Harmon et al., 1987). Despite the T. gallinae prefer pigeons and doves, but also can infect a wide range of bird families, such as bustards (Silvanose et al., 1998), psittacine birds (Baker, 1996) and fowl (Bushnell, 1942). Birds of prey (diurnal and nocturnal) are also frequently infected by the flagellated protozoan. Trichomonosis, characterised by necrotic ingluvitis, was first recognised as an emerging infectious disease of British finches in 2005 (Pennycott et al., 2005).

Researches on Avian trichomoniasis have showed a broad spectrum of virulence. With avirulent isolates, a bird may fail to even seroconvert after being inoculated with 1 X 106 organisms while with highly virulent isolates, a bird can subject to infection within 14 days after being inoculated with a single trichomonad, Furthermore, it has been demonstrated that clinically normal pigeons can harbor both avirulent and virulent isolates. However, uninfected doves and pigeons challenged with a mixture of virulent and avirulent isolates will subject to infection (Stabler, 1954).

Information on spread of $T$. gallinae found in Columbidae by different authors throughout the world vary greatly, ranging from $5.6 \%$ cited by Schulz et al., (2005) in mourning doves (Zenaida macroura) to $95 \%$ detected by Conti and Forrester (1981) in white-winged doves (Zenaida asiatica) in the USA. In Mosul city the research had been studied the pathological changes in digestive tract for domestic pigeons, the result showed that from 100 birds, $67 \%$ of them were appear a pathological lesions in digestive tract, most of the histopathological and gross lesions placed in intestine (29.3\%) followed by oropharynx $20.8 \%$, liver $16.6 \%$, esophagus $12.5 \%$, crop $10.4 \%$, proventriculus $6.2 \%$, and pancreas $4.2 \%$ (Saeed and Al-Nimma, 2010). A total of 100 free living urban pigeons were collected in Mousul city by Al- Sadi and Hamodi (2011), the overall prevalence were $16 \%$. Examination of Three species of pigeons were included in the study of Mousl city, they were stock dove (Columba oenas) 250, rock mountain dove (C. livia) 200, and collared doves (Streptopelia decaocto) 40, indicated prevalence rates of $22 \%, 17.5 \%$ and $10 \%$, for the three species respectively (AL-Bakry, 2009). In Diwaniya city the research had been studied the prevalence of $T$. gallinae in rock and domesticated pigeon, the results showed that from 65 rock pigeon and 130 domesticated pigeons, 20 and 60 birds were positive for the T. gallinae in the two type respectively, the total infection rate for the positive results were $30.7 \%$ and $46 \%$ for rock and domestic pigeons respectively (Sabeeh and AbdulAziz, 2012), the research in Diwaniya city have been studied the prevalence of the $T$. gallinae in doves Streptopelia decaocto and pigeons Columba livia, the results showed that from 100 doves and 100 pigeons, 13 and 29 birds were positive for T. gallinae in the two species respectively, the total infection rate $13 \%$ and $29 \%$ in the two type respectively (Abed, 2013).

Information on the avian trichomoniasis infection of pigeons in different region of Iraq appears to be poorly documented. The target of this research was to determine the spread of Avian trichomoniasis in three group of domestic pigeons as followed Al-zajal, Al-pakistani and Al-zuberi in Kirkuk city in northeast of Iraq, In addition of isolation, diagnosis and cultivation of $T$. gallinae.

\section{Materials and Methods}

\section{Area of study and Birds}

This study was carried out on three groups of domestic pigeons Columba livia domestica and these pigeons were purchased from pigeon fanciers in different area in Kirkuk city north eastern of Iraq Form a period middle August 2015 to middle April 2016. A total of 609 domestic pigeons were sampled, the management data were obtained about the pigeons before collecting the swab through personal interviews with the pigeon fanciers about the sex, and age of the pigeons. 


\section{Parasite}

The diagnosis of Trichomonas gallinae was confirmed by noting the parasites in direct wet mount preparations of specimens collected from mouth and esophagus using sterile cotton swab and put it in $0.5 \mathrm{ml}$ sterile normal saline concentration $0.9 \%$, and kept warm at $(20-30){ }^{\circ} \mathrm{C}$ until arrived to the laboratory then examined for motile protozoan within 30 minute, specimens were examined by light microscope under 10X and 40X magnification to detection jerky movement of trichomonads (Samour and Naldo, 2003).

\section{Staining method}

In current study we use three stains, namely Geimsa stain, Gram stain and for the first time fields stain. Smeared slides containing $T$. gallinae were dried in room temperature for (15-20) minute and stained using Gram stain following a standardized 4-step staining protocol with atom scientific (atom scientific company) Gram stain kits. Smeared slides containing $T$. gallinae were dried and fixed with methanol for $30 \mathrm{~s}$ and stained with Giemsa stain (Tasca and DeCarli, 2003) and allowed to air dry at room temperature for 15 to $20 \mathrm{~min}$.

The dried slides were subsequently rinsed with distilled water and allowed to dry at room temperature. The slides were observed under 100X and 40X magnifications. Parasites were stained with the Fields stain according to the procedure by Sivanandam and Mak (1975). Smeared slides containing $T$. gallinae were dried at room temperature for 15-20 minutes, and then, subsequently, added 8 drops of Field's stain B followed by 16 drops of Field's stain A on to the smear. Slides were then slightly shaking for $15 \mathrm{~s}$ before being rinsed for $2 \mathrm{~s}$ under a brisk stream of water, dried at room temperature. The slides were then observed under 100X and 40X magnifications.

\section{Cultivation method}

The positive sample of Trichomonas gallinae was cultivation in four media namely modified Diamond media (TYM) (Trypticase Yeast Extract media), CPLM media (Cysteine Pepton Maltose Media) and for the first time CM 161 media Oxoide trichomonas CM0161 and In-Pouch TV media. The initial oculation in the first three media was 2 X $105 \mathrm{cell} / \mathrm{mls}$ (estimation the number of trophozoites inculture made by haemocytometer and counted at x400 magnification), either in the In-Pouch TV media the initial culture was 1 X $105 \mathrm{cell} / \mathrm{mls}$. The Diamond media prepare according to Diamond (1957), and the CPLM media, CM 161 and In-Pouch TV media were commercially available. The CPLM media was purchased from Himedia (Indian company), CM 161 from Oxoide (UK company) and In-pouch TV media from (Biomed Diagnostics, US).

The $\mathrm{pH}$ of the culture media was adjusted to 6.5 with hydrochloric acid or sodium hydroxide $(\mathrm{NaOH})$ (Kharofa, 1999), the modified Diamond, CPLM and CM 161 media add to it Pencillin 100000IU/100mls, streptomycin $0.1 \mathrm{gm} / 100 \mathrm{mls}$ and nystatin $50000 \mathrm{IU} / 100$ mls. Modified Diamond and CPLM media add to it $10 \%$ of fresh inactive horse serum (inactivated it by boiling at $56^{\circ} \mathrm{C}$ for 30 minutes in water bath), and CM 161 add to it $8 \%$ of inactive horse serum. The inoculated In-tube and In-pouch culture media system were incubated at $370 \mathrm{C}$ in incubation and daily examination for six day, and the tubes were kept tightly capped and opened only for sampling. Only motile $T$. gallinae were counted.

\section{Results and Discussion}

The Wet mount preparation show the organism with pyriform in shape, which have four anterior flagella emerging from a belpharoplast and one recurrent flagellum stuck to an undulating membrane. $T$. gallinae also have a prominent axostyle at the posterior end of parasites, as it showed in figure 1.

Examination of the wet mount smear showed that $49.26 \%$ of domestic pigeons (300/609) were positive for T. gallinae, the highest infection rate was in Al-zajil group $64.92 \%$, and in Al-pakistani group pigeon the infectious rate were $50.98 \%$, while the low infection rate were recorded in zuberi group $30.41 \%$ as showed in table 1.

Giemsa stained cytoplasm with light purple and nucleus with dark purple, and clarification the flagella very well, as it showed in figure 2. Field's stained the nucleus and cytoplasm of $T$. gallinae dark pink and pink respectively as it showed in figure 3, and obvious the flagella, Gram stained the cytoplasm with light pink and nucleus with dark pink, but the flagella of T. gallinae is difficult to visualize with gram stain as it showed in figure 4 .

All media used in this research supported the growth and multiplication of organisms but at different rates. The InPouch medium which used for the first time for the cultivation of $T$. gallinae, the effect of this medium on the growth of $T$. gallinae in comparison to other culture media is presented in table 2 , the growth of $T$. gallinae 
increase to 72 hour reached to $1166000 \mathrm{cell} / \mathrm{mls}$ after inoculation with the initial inoculation $105 \mathrm{cell} / \mathrm{mls}$, InPouch culture system can kept the growth and motion till eight day, reach to the $50000 \mathrm{cell} / \mathrm{mls}$ in eight day after inoculation with normal motion.

Diamond medium gave the greatest growth till 48 hours, reached to 1325000 cell $/ \mathrm{mls}$, after cultivate with 2 X105 cell $/ \mathrm{mls}$ as an initial inoculation, then the number of growing cell decrease gradually after 48 hour, additionally the motion, and the isolation inter the death stage at 120 hour after inoculation where the number of survived cell was 0 cell $/ \mathrm{mls}$ at this time (Table 3 ).

CPLM medium gave the greatest growth till 24 hours reached to $1000000 \mathrm{cell} / \mathrm{mls}$ and the motion was active after inoculation with $2 \times 105 \mathrm{cell} / \mathrm{mls}$ as initial inoculation, then the number of growing cel decrease gradually after 24 hour additionally the motion, and the isolation stay alive till the 5 day after inoculation (Table $4)$.

Either CM 161 medium that used for first time to cultivation of $T$. gallinae in Iraq, this media gave the greatest growth till 48 hours after inoculation, with initial inoculation $2 X 105 \mathrm{cell} / \mathrm{mls}$, reached to $570000 \mathrm{cell} / \mathrm{mls}$ and the motion was active, after that the number of alive cell decease rapidly to $30000 \mathrm{cell} / \mathrm{mls}$ and $10000 \mathrm{cell} / \mathrm{mls}$ at 72 an 96 hour respectively, and the isolation enters the death stage at 120 hours after inoculation.

From the total of 609 Oro-pharyngeal swab smears 300 (49.26\%) were positive for present of T. gallinae in wet mount preparation, The traditionally Diagnosis of trichomonads was wet mount microscopy that based on microscopic diagnosis of motile trichomonads in the Oropharyngeal swab smears, Trichomonads can characteristic by the jerky movements in wet preparation, The sensitivity of the wet mount preparation differs from $38 \%$ to $82 \%$. There are two factors that wet mount preparation dependent on it, the first one is the sample ought to stay wet to keep the trichomonads viable and motile (the microscopic examination of wet preparation must be done within 30 minute after preparation of the wet smear) and the second factor is the examiner must be versed to recognizing trichomonads. Motility of trichomonads dependent on the temperature of the specimen. At room temperature in phosphate-buffered saline, the organism will remain alive for more than 6 hour however, the motility of the organisms becomes significantly attenuated at lower temperatures. Wet mount examination is clearly the most cost effective and simplicity diagnostic test, but the lack of sensitivity contributes to under diagnosis (DomeiKa et al., 2010). The wet mount preparation require at least $104 \mathrm{cell} / \mathrm{mL}$, furthermore incorrect temperature or too much time passing between take the sample and assessment results in loss of motility making difficulty to diagnosis of trichomonads from epithelial cell or lymphocytes that present in the specimen (Garber, 2005).

Columbidae are known as the $T$. gallinae main host, especially the domestic pigeon (Columba livia), which has been believed to be responsible for the world-wide prevalence of parasite. only limited surveys of $T$. gallinae infection in domestic pigeons have been reported throughout the world, The prevalence of $T$. gallinae in current study in domestic pigeons is (49.26\%) and this rate was close to that recored by Sabeeh and AbdulAziz, (2012) reached to $46 \%$ in Diwaniya city in domestic pigeon, either Sansano-Maestre et al (2009) recorded $44.8 \%$ in spain. AL-Bakry, (2009) examination of Three species of pigeon, they were stock dove (Columba oenas) 250, rock mountain dove (C. livia) 200, and collared doves (Streptopelia decaocto) 40, indicated prevalence rates of $22 \%, 17.5 \%$ and $10 \%$, for the three species respectively in Mousl city, the current study recorded the low infectious rate from that recorded by Al-Safar (2014) reached to $61.32 \%$ in the domestic pigeon in Baghdad city, But invasion rate to T. gallinae in world are very different, The variation in prevalence of $T$. gallinae found in pigeons might be due to many factors like climatic conditions, geographical difference, seasonas of sampling, resistance of the host, different feeding habits or feed pattern of the birds, age of birds, difference in housing conditions, husbandry practices in different regions, and detection assay.

The Field's staining method was used in this study following the protocol of Sivanandam and Mak (1974) with a slight modification of timing where the original 10 to $12 \mathrm{~s}$ for malarial staining was extended to 15 to $20 \mathrm{~s}$ in the study by Afzan et al., (2010) to optimize staining results for detecting $T$. gallinae. Castillo and Rojas (1997) reported that Field's stain was more sensitive than Giemsa (78.7\% versus70.5\%), to diagnosis of American cutaneous leishmaniasis. While Afzan et al., (2010) reported that both Giemsa and Field's stain are equally good for detecting $T$. vaginalis. In the present study, the results suggest that Giemsa stain more efficiency from Field's stain to detecting T. gallinae, may be because of the short time of staining in field's stain. The staining procedure for Field's stain required only $20 \mathrm{~s}$ in the present study as opposed to $20 \mathrm{~min}$ with Giemsa 
staining. However, Field's stain has several advantages compared to Giemsa stain such as the ease and rapidity in preparation, making it favorable stain for both field and laboratory use. Gram stain is a widely used method for identification of some bacteria such as Neisseria gonorrhoeae, Chlamydia trachomatis, Mycoplasma hominis, Ureaplasma urealyticum and fungi (Barberis et al., 2002), Harrington and Williams (1999) however, did report that Gram-stained T. vaginalis require skilled expertise and confidence for detection. This observation of theirs concurred with in our finding the gram stain gave the low efficiency to staining $T$. gallinae compared to other stain we use in current study, although Gram stain characteristics between the nucleus and cytoplasm but it wa poor stain the flagella, The efficiency of Giemsa and field's stain due to they were obtained commercially in powder form, whereas Gram stain is usually commercially available in solution form. Powder forms have longer shelf-life (Afzan et al., 2010).

Cultivation in liquid medium has stayed the gold standard method for diagnosis of Trichomoniasis from the 40 past years ago. The regularity broth used is Diamond's medium (Petrin et al., 1998). Even when broth cultures are mixed with antibiotics to decrease contamination by normal flora of the swabbing sites, the major problem in cultivation of trichomonads is control of the contamination by the bacteria. Cultivation of trichomonads are the most effective method to making right epidemiology of trichomonads species, but the culture wasn't easily available in many center, to resolve these issue, they developed the system like In-pouch TV culture (BioMed Diagnostics, USA) where they can inoculated the sample in a two chambered bag, that allow the examiner to use the pouches as wet mount preparation and immediate observation of trichomonads through the pouch bags that use as slide after inoculation with the sample, or can incubation the inoculated pouches to diagnosis the trichomonads by cultivation (Borchardt and Smith., 1991).

Trichomonas gallinae is a protozoan that grows very slowly under aerobic conditions. Trichomonas $s p$. grown in the bottom of the tubes or pouch, the pouches should be incubated in a vertical position. The use of In-Pouch TV culture system for cultivation of trichomonads were close efficiency to Diamond's medium in glass (Borchardt et al., 1997). Each test pouch have two chambers, that separated by a narrow channel and this channel allow to pass the fluid between the two chamber. The lower chamber consist of modified Diamonds medium, some of liquid should be squeezed from the lower chamber to upper chamber before sample inoculation, and the sample obtained by a cotton swab, if desired the inoculated pouches can be examined microscopically as direct wet mount preparation for motile trichomonads. The inoculation pouch incubated and daily examined for a week by held in a plastic viewer. The In-pouch culture system was more sensitivity from wet mount preparation to diagnosis of the trichomonads, and have a higher sensitivity comparable to In-tube culture system media, the greatest advantage of this method is that trichomonads stay survival up to 7 days after inoculation by the parasite's, and that we found in the present research. In current study we used In-pouch TV culture system to grow $T$. gallinae for first time, and this medium gave the highest support to $T$. gallinae even to 8 day after inoculation. The additional advantage of the In-pouch TV test has a relatively long shelf life (6 months) in room temperature, In-Pouch TV culture system provide to be superior to Intube culture system, and can support the growth of $T$. gallinae even with rare number of Trichomonads.

In-Pouch medium had several special advantages such as preventing the contamination by observation of the inoculation media can be made directly through the chamber that can use it as the slide and take it in the stage of the microscope. and the In-pouch test easily transported from the site of sampling to the laboratory, and pouches teast can kept in room temperature, other In-tube culture media that prepared need refrigeration, furthermore the cost of In-pouch culture system is less than the cost of prepare culture media, in conclusion the In-pouch culture media should be used as a routine procedure to diagnosis of Trichomonas sp. Modified Diamond's evolved from Diamond's (TYM) medium, originally developed by Diamond in 1957. The main ingredients were trypticase digest, yeast extract, cysteine, maltose, ascorbic acid, and sheep serum. Several modifications of this medium have been made, and Diamond later developed several other forms of media for other protozoans (Diamond, 1968). All of these were used for propagation of Trichomonads and were called modified Diamond's. In 1980, Klaas, at the Centers for Disease Control, returned to the original Diamond formulation and made the following changes: horse serum replaced sheep serum; the concentrations of maltose, cysteine, and ascorbic acid were increased; agar was eliminated; and antibiotics were added to suppress the growth of bacteria and fungi (Fouts and Kraus, 1980), in present research Diamond medium support the growth of $T$. gallinae as In-pouch and more than the other two In-tube culture media, 
Table.1 Infection rate between different pigeon group

\begin{tabular}{|l|l|l|l|l|}
\hline Pigeon group & $\begin{array}{l}\text { number of } \\
\text { tested birds }\end{array}$ & $\begin{array}{l}\text { Number of } \\
\text { intact birds }\end{array}$ & $\begin{array}{l}\text { Number of } \\
\text { infection birds }\end{array}$ & $\begin{array}{l}\text { Infectious } \\
\text { retio \% }\end{array}$ \\
\hline Al-zajle & 211 & 74 & 137 & $64.92 \%$ \\
\hline Al-pakistani & 204 & 100 & 104 & $50.98 \%$ \\
\hline Al-Zuberi & 194 & 135 & 59 & $30.41 \%$ \\
\hline total & 609 & 309 & 300 & $49.26 \%$ \\
\hline Statically analysis & $\begin{array}{l}\mathrm{X}^{2}=48.540 \\
\mathrm{P}-\mathrm{v}=0.0008\end{array}$ & $* * \mathrm{P}<0.01$ \\
\hline
\end{tabular}

Table. 2 Showed the average and motion of the T. gallinae in In-Pouch medium $3 \mathrm{ml}$

\begin{tabular}{|l|l|l|l|l|l|l|l|l|l|l|}
\hline Concentration & \multicolumn{2}{|l|}{24 hour } & \multicolumn{2}{l|}{48 hour } & \multicolumn{2}{l|}{72 hour } & \multicolumn{2}{l|}{96 hour } & \multicolumn{2}{l|}{120 hour } \\
\cline { 2 - 9 } & Motion & Number & Motion & Number & Motion & Number & Motion & Number & Motion & Number \\
\hline $100 \%$ & Normal & 383000 & Active & 800000 & $\begin{array}{l}\text { Very } \\
\text { active }\end{array}$ & 1166000 & Active & 600000 & Normal & 33000 \\
\hline
\end{tabular}

Table.3 Showed the average and motion of the T. gallinae in modified Diamond medium $10 \mathrm{ml}$

\begin{tabular}{|l|l|l|l|l|l|l|l|l|l|l|}
\hline Concentration & \multicolumn{2}{|l|}{24 hour } & \multicolumn{2}{l|}{48 hour } & \multicolumn{2}{l|}{72 hour } & \multicolumn{2}{l|}{96 hour } & \multicolumn{2}{l|}{120 hour } \\
\cline { 2 - 9 } & Motion & Number & Motion & Number & Motion & Number & Motion & Number & Motion & Number \\
\hline \multirow{2}{*}{$100 \%$} & Normal & 691000 & Active & 1325000 & Weak & 216000 & $\begin{array}{l}\text { Very } \\
\text { weak }\end{array}$ & 25000 & $\begin{array}{l}\text { Death } \\
\text { stage }\end{array}$ & 0 \\
\hline
\end{tabular}

Table.4 Showed the average and motion of the T. gallinae in CPLM medium $10 \mathrm{ml}$

\begin{tabular}{|l|l|l|l|l|l|l|l|l|l|l|}
\hline Concentration & 24 hour & \multicolumn{2}{|l|}{48 hour } & \multicolumn{2}{l|}{72 hour } & \multicolumn{2}{l|}{96 hour } & \multicolumn{2}{l|}{120 hour } \\
\cline { 2 - 9 } & Motion & Number & Motion & Number & Motion & Number & Motion & Number & Motion & Number \\
\hline \multirow{2}{*}{$100 \%$} & Active & 1000000 & Normal & 550000 & Weak & 250000 & $\begin{array}{l}\text { Very } \\
\text { weak }\end{array}$ & 200000 & $\begin{array}{l}\text { Death } \\
\text { stage }\end{array}$ & 100000 \\
\hline
\end{tabular}

Table.5 Showed the average and motion of the T. gallinae in CM 161 medium $10 \mathrm{ml}$

\begin{tabular}{|l|l|l|l|l|l|l|l|l|l|l|}
\hline Concentration & \multicolumn{3}{|l|}{24 hour } & 48 hour & \multicolumn{2}{l|}{72 hour } & \multicolumn{2}{l|}{96 hour } & \multicolumn{2}{|l|}{120 hour } \\
\cline { 2 - 9 } & Motion & Number & Motion & Number & Motion & Number & Motion & Number & Motion & Number \\
\hline \multirow{2}{*}{$100 \%$} & Normal & 470000 & Active & 570000 & Weak & 30000 & $\begin{array}{l}\text { Very } \\
\text { weak }\end{array}$ & 10000 & $\begin{array}{l}\text { Death } \\
\text { stage }\end{array}$ & 0 \\
\hline
\end{tabular}


Fig.1 Trichomonas gallinae in wet mount preparation under 40X magnification

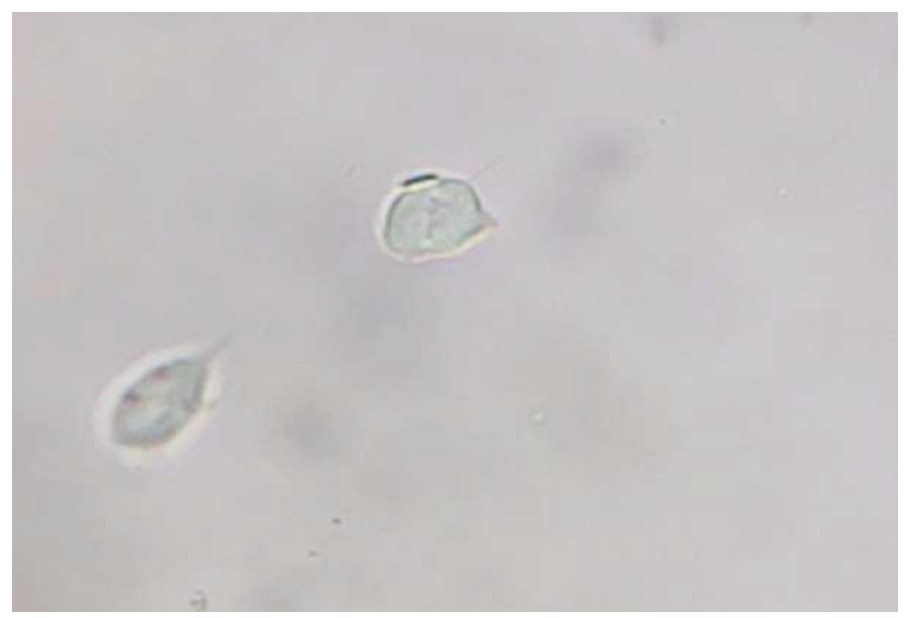

Fig.2 Trichomonas gallinae stained with Giemza stain under 100X magnification

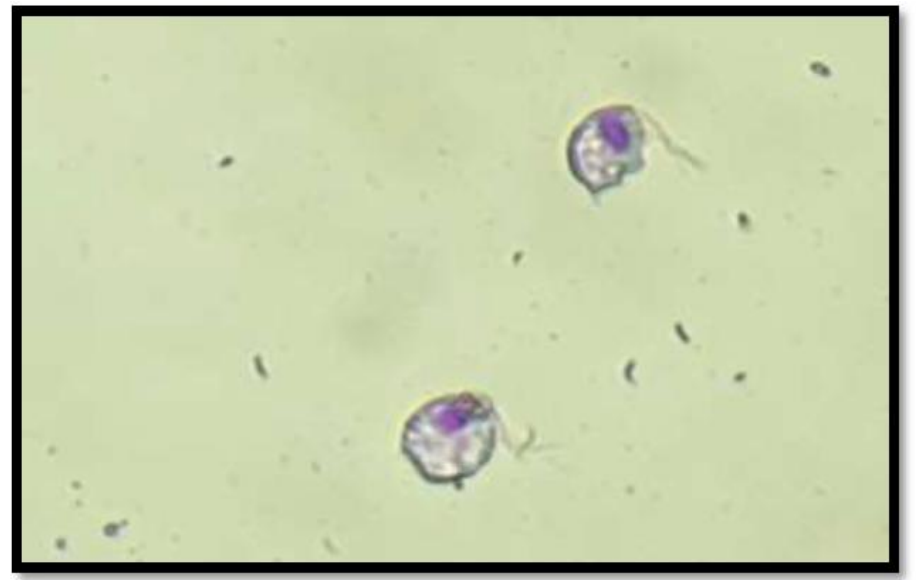

Fig.3 Trichomonas gallinae stained with Gram stain under 100X magnification

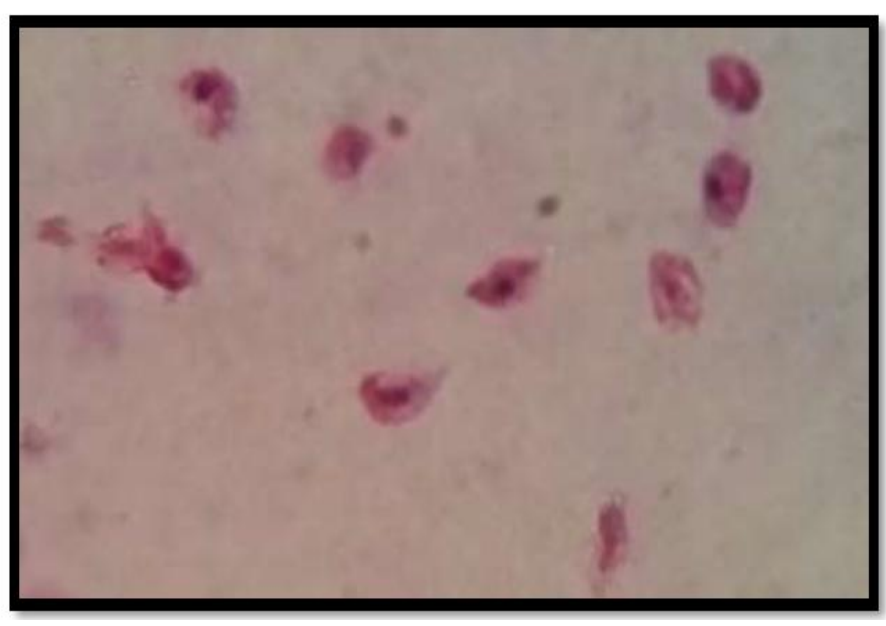


Fig.4 Trichomonas gallinae stained with Field's stain under 100X magnification

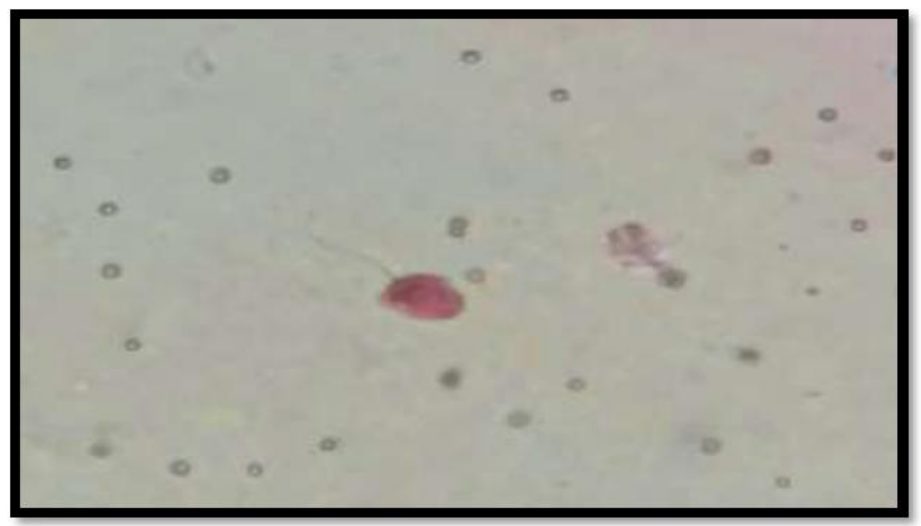

Diamond medium gave the growth to 48 hour then decrease the growth gradually, in five day after inoculation the isolation in culture media enter the death stage, and not found any motile cell in this time. In-Pouch TV medium was more significant than Modified Diamoind. CPLM and CM 161 media to support the growth of $T$. gallinae, this result was del with Borchardt et al., (1997).

In current study the CM 161 used for first time to cultivation of T. gallinae in Iraq, the growth in CM 161 was acute decrease after 48 hour the reason may be due to that other prepared media add to it $10 \%$ of inactive horse serum while CM 161 add to it $8 \%$ inactive horse serum this result del with Gelbart et al., (1990) that grew T. gallinae in Modified Diamond's medium uses $12 \%$ horse serum, while Kupferberg's contains only 5\% bovine serum. The additional serum may be protective, diminishing the catastrophic lag phase that occurs when clinical specimens are inoculated into media. Increasing the serum concentration in CM 161 might support the growth of $T$. gallinae as other media we used.

In this research the CPLM medium allows more prolific growth over a shorter period of time through the first 24 hours this result del with Al-Dabbagh and Kharofa (2007), the growth of $T$. gallinae decrease after 24 hour, and the motion be weak, but some trichomonds stay alive and very weak motion to 5 day after inoculation.

\section{References}

Abed, A.A.A. 2013. The incidence of Trichomonas in the dove (Streptopelia decaocto) and pigeon (Columba livia) at Al-Diwaniya city and its effects on blood picture, Coll. of Vet. Med. Unive. of Al-Qadissiya, AlQadissiya J. Vet. Sci. med., 12(1) p: 72-77.

Afzan, M.Y., Sivanandam, S. and Kumar, G.S. 2010. Modified Field's staining a rapid stain for Trichomonas vaginalis, Diagn. Microbiol. J., 68(2): 159-162.
Al-Bakry, H.S. 2009. Prevalence of avian trichomoniasis in different species of pigeons in Mosul, Iraq, Iraq $J$ Vet. Sci., 23(2): 105-109.

AL-Dabbagh, N.Y. and Karofa, A. 2007. Prepration and evaluation of local medium for culture and growth of Trichomonas vaginalis isolated from patients in Mosaul, Iraq. J. Biotech., 6(2): 111-119.

Al-Sadi, H.I. and Al-Hamodi, A.Z. 2011. Prevalence and Pathology of Trichomoniasis in Free Living Urban Pigeons in the City of Mosul, Iraq, Vet. World, Vol. 4(1): 12-14.

Al-saffar, E.H.J. 2014. Epidemiology and pathology of Trichomonas gallinae in wild and domestic pigeons in Baghdad city, Master thesis.

Baker, J.R. 1996. Causes of mortality and morbidity in exhibition budgerigars in the United Kingdom. Vet. Record, 139: 156-162. doi: 10.1136/vr.139.7.156.

Barberis, I.L., Pájaro, M.C., Godino, S., Pascual, L., Daniele, M. 2002. Diagnóstico 187 microbiológico de vaginosis bacteriana (Microbiological diagnosis of 188 bacterial vaginosis. Acta Bioquim Clin Latinoam, 36: 541-545.

Borchardt, K.A., Zhang, M.Z., Shing, H., Flink, K., A. 1997. Comparison of the sensitivity of the In_Pouch TV, Diamond's, and Trichosel media for detection of Trichomonas vaginalis, Genitourin Med., 73: 297-298.

Borchardt, K.A. and Smith, R.F. 1991. An evaluations of an inpouch TV culture method for diagnosis Trichomonas vaginalis infection, Gentourin. Med., 67: 49-52.

Bushnell, L.D. 1942. Trichomoniasis of the upper digestive tract of domestic birds, Poultry Sci., 21: 466-467.

Bunbury, N., Jones, C.G., Greenwood, A.G., Bell, D.J. 2007. Trichomonas gallinae in Mauritiancolumbids: implications for an endangered endemic. J. Wildlife Dis., 43: 399-407.

Castillo, C.M., Rojas, C. 1997. Evaluation of popular stains for the diagnosis of American cutaneous leishmaniasis. Mem Inst Oswaldo Cruz, Rio de Janeiro 92: 531-532. 
Conti, J.A. and Forrester, D.J. 1981. Inter relationships of parasites of white-winged doves andmourning doves in florida. J. Wildlife Dis., 17: 529-536.

Diamond, L.S. 1957. The establishment of various trichomonads of animals and man in axenic culture. $J$. Parasitol., 43: 488-490.

Diamond, L.S. 1968. Techniques of axenic cultivation of Entamoeba histolytica Schaudinn 1903 and E. histolytica-like amoebae. J. Parasitol., 54: 1047-1056. 4.

Domeika, M., Zhurauskaya, L., Savicheva, A., Frigo, N., Sokolovskiy, E., A Hallen, A., Unemo, M., Ballard, R.C. and E.E. SRH Networka. 2010. Guidelines for the laboratory diagnosis of trichomoniasis in East European countries, JEADV, 24: 1125-113.

Fouts, A.C., and S.J. Kraus. 1980. Trichomonas vaginalis: reevaluation of its clinical presentation and laboratory diagnosis. J. Infect. Dis., 141: 137-143.

Garber, G.E. 2005. the laboratory diagnosis of Trichomonas vaginalis. Can. J. Infect. Dis. Med. Microbiol., 16(1): 35-8.

Gelbart, S.M., Thomason, J.L., Osypowski, P.J., Kellett, A.V., James, J.A., and Broekhuizen, F.F. 1990. Growth of Trichomonas vaginalis in commercial culture media, J. Clin. Microbiol., 28(5): 962-964.

Grabensteiner, E., Bilic, I., Kolbe, T., Hess, M. 2010. Molecular analysis of clonal trichomonad isolates indicates the existence of heterogenic species present in different birds and within the same host. Vet. Parasitol., 172: 53-64.

Harmon, W.M., W.A. Clark, A.C. Hawbecker, and M. Stafford. 1987. Trichomonas gallinae in Columbiform birds from the Galapagos Islands. J. Wildl. Dis., 23: 492-494.

Harrington, B.J., Williams, D.L. 1999. Recognizing trichomonads in Gram- 206 stained smears. Lab. Med., 30: 803-806.

Kharofa, W.A. 1999. An epidemiological study and cultivation of Trichomonas vaginalis in Mosul City. M.Sc. Thesis, College of Science University of Mosul.

Mesa, C.P., R.M. Stabler and M. Berthrong. 1961. Histopathological changes in the domestic pigeon infected with Trichomonas gallinae (Jones' barn strain. Avian Dis., 5: 48-60.

Narcisi, E.M., Sevolan, M. and Honigberg, B.M. 1991. Pathologic changes in pigeons infected with a virulent
Trichomonas gallinae strain (Eigberg. Avian Dis., 35, 55-61.

Pertin, D., Delgaty, K., Bhatt, R. and Garber, G. 1998. Clinical and Microbiological aspects of Trichomonas vaginalis. Clin. Microbiol. Rew., 2(I): 300 - 317.

Pennycott, T.W., Lawson, B., Cunningham, A.A., Simpson, V., Chantrey, J. 2005. Necrotic ingluvitis in wild finches, Vet. Rec., 157: 360.

Sabeeh, N. and AbdulAziz, A. 2012. Isolation of birds Trichomoniasis from domesticated and wild pigeon at Al-Diwaniya city, Al-Qadissiya J. Vet. Sci. Med., 11(1): 101-104.

Saeed, M.G. and Al-Nimma, A.A. 2010. Study of pathological changes in digestive system of domestic pigeons (Columba livia) in Mosul city, Iraqi J. Vet. Sci., 24(2): 115- 21.

Samour, J.H. and Naldo, J.L. 2003. Diagnosis and therapeutic management of Trichomoniasis in falcons in Saudi Arabia. J. Avian Med. Surgery, 17(3): 136143.

Sansano-Maestre, J., Garijo-Toledo, M.M. and GomezMunoz, M.T. 2009. Prevalence and genotyping of Trichomonas gallinae in pigeons and birds of prey. Avian Pathol., 38(3): 201- 207.

Schulz, J.H., A.J. Bermudez, and J.J. Millspaugh. 2005. Monitoring presence and annual variation of trichomoniasis in mourning doves. Avian Dis., 49: 387-389.

Silvanose, C.D., Samour, J.H., Naldo, J.L. and Bailey, T.A. 1998. Oropharyngeal protozoa in captive bustards: clinical and pathological considerations. Avian Pathol., 27: 226_230.

Sivanandam, S., and Mak, J.W. 1974. Various Giemsa preparations and their use in filariasis. J. Med. Health Lab. Technol. Malays., 1: 9-11.

Sivanandam, S., and Mak, J.W. 1975. Some problems associated with the 243 processing and staining of blood films for filarial diagnosis. J. Med. 244 Health Lab. Technol. Malays., 2: 1-2.

Stabler, R.M. 1954. Trichomonas gallinae: A reveiw. Exper. Parasitol., 3: $368-402$.

Tasca, T. and De Carli, G.A. 1999. Prevalence of Trichomonas gallinae from the upper digestive tract of the common pigeon (Columba livia) in the southern Brazilian state, Rio Grande do Sul, Parasitologia al Dia, 23: 42-43.

\section{How to cite this article:}

Sadia Sh. Hamad and Hazha H. Hassan. 2017. Isolation, Diagnosis and Cultivation of Trichomonas gallinae from Domestic Pigeon in Kirkuk City, Iraq. Int.J.Curr.Res.Aca.Rev. 5(2), 10-18.

doi: http://dx.doi.org/10.20546/ijcrar.2017.502.002 